\title{
The role of retinoic acid signaling in starfish metamorphosis
}

\author{
Shumpei Yamakawa* (10, Yoshiaki Morino, Masanao Honda and Hiroshi Wada
}

\begin{abstract}
Background: Although retinoic acid (RA) signaling plays a crucial role in the body patterning of chordates, its function in non-chordate invertebrates, other than its mediation of environmental cues triggering metamorphosis in cnidarians, is largely unknown. We investigated the role of RA signaling in the metamorphosis of starfish (Echinodermata).

Results: We found that exogenous RA treatment induced metamorphosis in starfish larvae. In contrast, inhibitors of RA synthesis and RA receptors suppressed metamorphosis triggered by attachment to a substrate. Gene expressions of the RA signaling component were detected in competent larvae.

Conclusions: This study provides insight into the ancestral function of RA signaling, which is conserved in the metamorphosis of cnidarians and starfish.
\end{abstract}

Keywords: Retinoic acid signaling, Metamorphosis, Echinoderm, Starfish

\section{Background}

Retinoic acid (RA) plays a critical role in the body patterning of chordates, such as the anterior-posterior patterning of the central nervous system and pharyngeal arches [1-3]. RA synthesized by retinal dehydrogenase (Raldh) regulates downstream gene expression through binding to the retinoic acid receptor (RAR) and the retinoid $\mathrm{x}$ receptor (RXR) heterodimer nuclear receptor. Although RXR was identified in various metazoan taxa including cnidarian, arthropod, and nematode [4], Raldh and RAR, as well as cytochrome P450 26 (CYP26), which degrades RA, had been described only in chordates, and thus RA signaling was thought to be specific to chordates, and the acquisition of the gene families for RA signaling was thought to be a key step in the evolution of the chordate body plan [3]. However, Cañestro et al. reported that gene families of Raldh, RAR, and CYP26 are encoded in the genomes of non-chordate deuterostomes [5]. Following additional genomic surveys of other invertebrates, the origin of RA signaling was pushed back to the common ancestor of metazoans $[6,7]$.

\footnotetext{
*Correspondence: shumpei.yamakawa@gmail.com Graduate School of Life and Environmental Sciences, University of Tsukuba, Tsukuba, Ibaraki 305-8572, Japan
}

Despite these genomic surveys, the function of RA signaling in non-chordate deuterostomes remains largely unknown, other than the observation of pseudopodial cable growth on micromere-delivered cells from Hemicentrotus pulcherrimus after RA treatment [8]. Sciarrino et al. reported that RA treatment on Paracentrotus lividus did not induce specific phenotype except for delaying development [9]. Although Marlétaz et al. suggested that the promoter region of sea urchin Hox genes had an RA response element [3], no additional evidence of the effect of RA on Hox gene expression has been reported.

Insight into the ancestral function of RA signaling can be gained from the study of cnidarians, a basal lineage of animals. Fuchs et al. showed that RA signaling is involved in the life cycle transition of cnidarians [10]. Medusozoan cnidarians have two distinct life cycle phases. Fertilized eggs first develop into planula larvae and then, through further development, become sessile polyps. When the polyps receive environmental stimuli, such as a temperature change, they metamorphose into medusae [10]. Fuchs et al. reported that RA is an internal regulator of this process and suggested that RA works through binding with RXR [10]. Because RXR is known to be involved in the metamorphosis of insects and frogs [11-16], RA 
signaling was suggested to also have a role in the life cycle regulation of metazoans.

Molecular mechanisms for metamorphosis have been investigated in echinoderms, primarily echinoids [1728]. In the sea urchin Strongylocentrotus purpuratus, larvae acquired competence to metamorphose 4.5-6 weeks after fertilization [25] and commenced metamorphosis in response to substrates such as algal sheets [29]. Previous studies suggested that thyroid hormone $(\mathrm{TH})$ and histamine (HA) functioned as modulators of larval growth and the competent state [19-21, 24-27]. Although several works also revealed nitric oxide (NO) signaling functioned as a negative regulator upon settlement [17, 18], any role of RA signaling in metamorphosis is not reported in any taxa of echinoderm.

Here, we investigate the role of RA signaling in asteroid metamorphosis. We used the starfish Patiria pectinifera, as it acquires competence to metamorphose earlier than sea urchin species (about 1 week after fertilization). We show that exogenous RA treatment induced larval body absorption and juvenile rudiment development in competent larvae. Conversely, the inhibition of endogenous RA synthesis or binding of RA to RAR blocked metamorphosis. In addition, genes related to RA signaling were expressed in the juvenile and larval stages. These results suggest that RA signaling acts as a regulator of metamorphosis after settlement.

\section{Methods}

\section{Sampling and culture}

We collected adult specimens of $P$. pectinifera around the mainland of Japan: Manaduru (Kanagawa Prefecture), Asamushi (Aomori Prefecture), and Hiraiso (Ibaraki Prefecture). Fertilization and embryo rearing were performed from described previously [30]. From 2 days post-fertilization (dpf), the larvae were fed Chaetoceros calcitrans (purchased as Sun-culture: Marine Tec, Aichi) by rearing in seawater with $50,000 \mathrm{cells} / \mathrm{ml}$. We changed seawater with fresh algae every other day.

\section{Reagent treatment}

The RA signaling pathway was activated by exogenous all-trans RA (Sigma-Aldrich, St Louis, CAS number: 30279-4). RA signaling was inhibited by the Raldh inhibitor $\mathrm{N}$, N-diethylaminobenzaldehyde (DEAB, Tokyo Chemical Industry, Tokyo, CAS number: 120-21-8) or the RAR antagonist RO41-5253 (RO, Focus Biomolecules, Pennsylvania, CAS number: 144092-31-9). We prepared $100 \mathrm{mM}, 1 \mathrm{M}$, and $50 \mathrm{mM}$ stocks of RA, DEAB and RO, respectively, in dimethyl sulfoxide (DMSO). Larvae were incubated in $2 \mathrm{ml}$ artificial seawater containing $2 \mu \mathrm{l}$ of reagents or DMSO in 12 -well plates at $22{ }^{\circ} \mathrm{C}$. For the treatment without a substrate, 10 larvae were incubated in one well. In the experiments conducted to induce metamorphosis with a substrate, a single larva was cultured in one well to identify individuals. We observed metamorphosis proceed in the same manner in these densities of larvae. Coral sand from tanks of adult $P$. pectinifera was used as a substrate for $P$. pectinifera experiments [31]. For cases in which drug treatment continued for more than 2 days, we changed the seawater with the same concentration of drugs every other day.

We considered the ability to induce metamorphosis to have been achieved when larval body absorption and the development of juvenile rudiment with primary podia were observed (Fig. 1). We considered larvae adhered to the substrate with brachiolar arms to be settled. In order to test effects of those reagent, we set several conditions in the experiments and assumed these conditions as main factors: density of DEAB $(0,100$ and $300 \mu \mathrm{M})$ and presence/absence of $\mathrm{RO}(1 \mu \mathrm{M})$ for settlement and metamorphosis, and combination of density of RA $(0.1$ and $1 \mu \mathrm{M})$ and presence/absence of RO $(3 \mu \mathrm{M})$ for metamorphosis. Each data was obtained from three batches of different parent pair. Within batches, treatments of density of $\mathrm{DEAB}$ and presence/absence of $\mathrm{RO}$ were repeated thrice and twice, respectively. In total, we used 324 and 324 individuals for experiments of density of $D E A B$ in settlement and metamorphosis, respectively, 144 and 144 for those of presence/absence of RO in settlement and metamorphosis, respectively, and 240 for those of combination of density of RA and presence/absence of $\mathrm{RO}$ in metamorphosis.

\section{Statistical analysis}

The metamorphosis ratio was calculated by dividing the number of metamorphosis commenced larvae by the number of treated larvae. In the case of reagent treatment with substrate, metamorphosis ratio was calculated by dividing the number of metamorphosed larvae by the number of settled larvae.

We used ANOVA to evaluate differences of reagent treatments on the metamorphosis or settlement, assuming above conditions and batches as main factors and "blocks," respectively, because preliminary tests in some treatments revealed slight, but not essential, differences among batches. If we had two factors, we assessed an interaction of the two factors. Also, multiple comparison was performed by Tukey's honestly significant difference test. The R statistical software was used for all statistical analyses [32].

As to the assumption of normal distribution, we use Kolmogorov-Smirnov test and confirmed no significant difference from the assumption of normality. Also, Bartlett's test was used to test homogeneity of variances prior to ANOVA. In a few experiments, we found significant 


\section{Competent Larva}

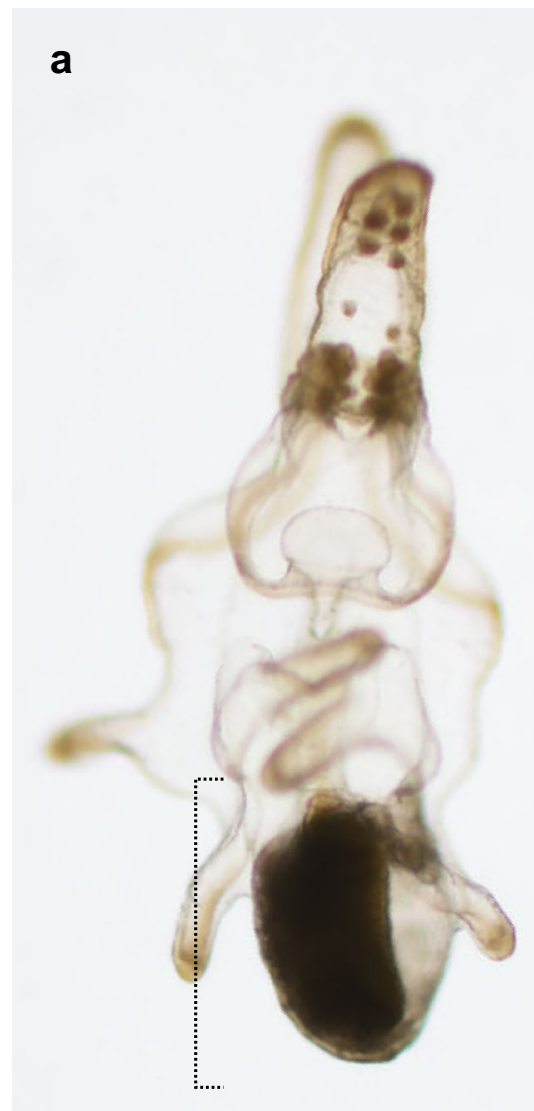

b

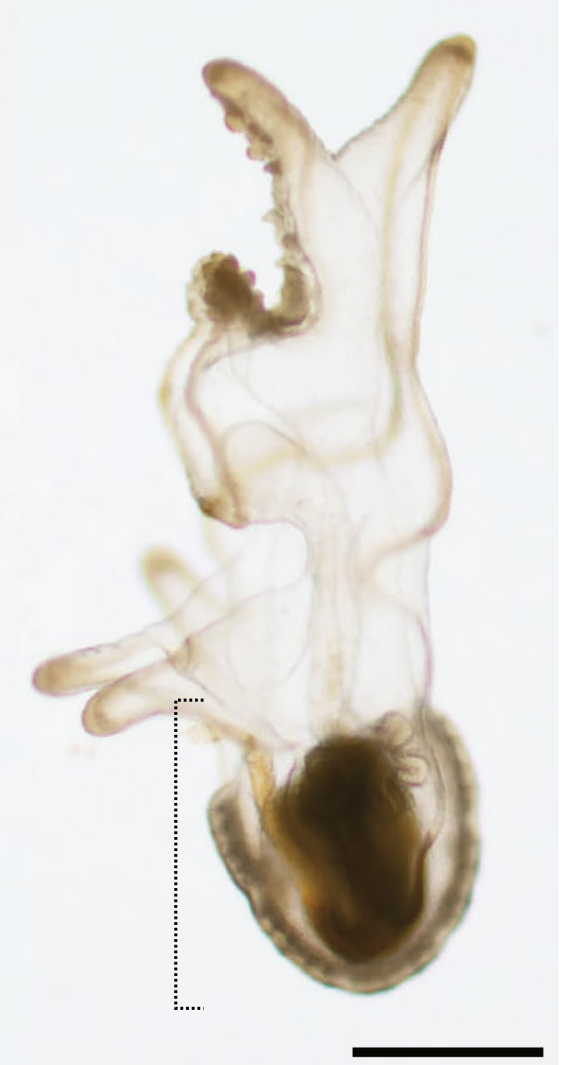

Metamorphosis
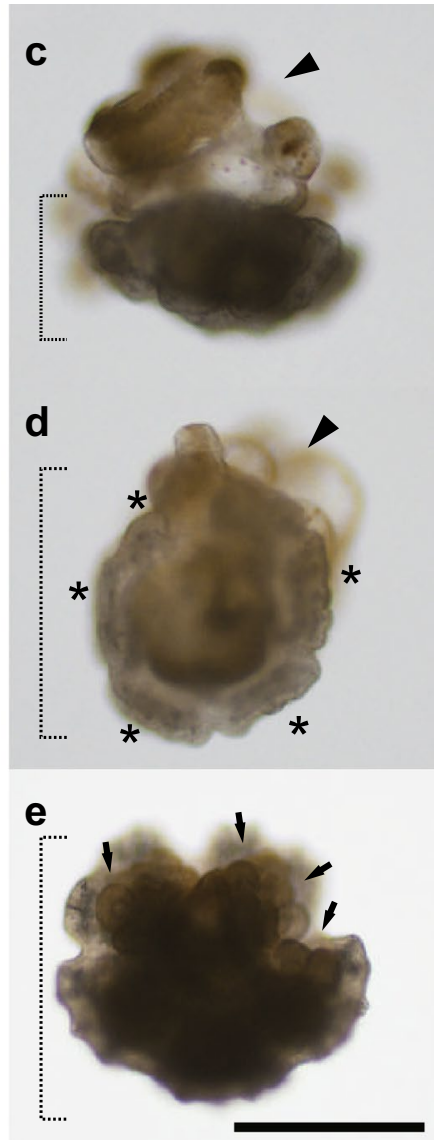

Fig. 1 Competent and metamorphosis larva of P. pectinifera. a, b Ventral (a) and left side (b) view of competent 20-dpf larva. c-e Metamorphosis larva in $24 \mathrm{~h}$ after settlement. c Larval ventral side of metamorphosis larva. $\mathbf{d}$, e attend on aboral and oral side of juvenile rudiment, respectively. Dot lines indicate juvenile rudiment and asterisk marks do developed juvenile arms. Arrow heads point absorbed larval body and arrows do primary

podia. Size of scale bar is $250 \mu \mathrm{m}$

violations of the equal variance assumption and conducted Welch's one-way ANOVA to test main effects without the assumption of equality. However, there were no differences in statistical conclusions between the main factor of ANOVA and the Welch's method. We thus used ANOVA with assumption of equal variance in the following analyses because we needed multi-dimensional analyses simultaneously and because ANOVA is robust to deviations from equal variance, especially if numbers of sample are equal [33], and normality assumptions [34].

\section{Identification of genes related to RA signaling in starfish}

To gather sequence of RA signaling related genes, we performed transcriptome analysis on samples of blastula (13 h post-fertilization), brachiolaria larva (14 dpf) and adult epidermis in starfish $P$. pectinifera. Each sample was collected from three biological replicates: The samples of blastula and brachiolaria larva were collected from progeny of three distinct pairs of parents, and the adult epidermis was collected from three mother specimens. Total RNA was extracted using TRIzol reagent (Life Technologies, Carlsbad, CA, USA) and treated with DNase and cleaned up using the RNeasy kit (Qiagen, Hilden, Germany). Paired-end libraries (200 bp) were prepared using a TruSeq RNA Sample Preparation Kit (Illumina). Sequencing on the Hiseq 2000 platform (Illumina, San Diego, CA, USA) was performed at the National Institute of Basic Biology (NIBB) The raw reads were deposited in the DDBJ Sequence Reads Archives (DRA006662). The low-quality reads were filtered using the NGS QC Toolkit57 [35]. De novo assembly was conducted by Trinity58 (version: trinityrnaseq_r20140717) [36]. 
We recovered nucleotide sequences coding for raldh, rar and rxr from gene models assembled from our de novo transcriptome data of larval stages for $P$. pectinifera. The orthologies of these genes were confirmed by molecular phylogenetic analysis (Additional file 1: Fig. S1, Additional file 2: Fig. S2). All sequences are shown in Additional file 3: Table S1 and aligned using MAFFT ver. 7 (https://mafft.cbrc.jp/alignment/server/) with the default parameters [37]. Amino acid sites for tree construction were selected by trimAl using a gap threshold value of 0.8 [38]. A best-fitting amino acid substitution model and a maximum likelihood tree were inferred using RAxML 8.2.0 [39]. Confidence values were calculated after 1000 bootstrap runs.

\section{Whole-mount in situ hybridization}

cDNA was obtained by reverse transcription from larval RNA using PrimeScript 1st strand cDNA Synthesis kit (Takara, Shiga, Japan). Additional file 4: Table S2 shows the sequences of the primers used for the amplification of raldha, raldhb, raldhc, rar, and rxr. We used 40-bp reverse primers including a 20-bp T3 promoter sequence to synthesize Dig-labelled RNA probes for in situ hybridization. Dig-labelled RNA probes for raldha, raldhb, raldhc, rar, and $r x r$ were transcribed from PCR products using T3 RNA polymerase (Roche, Mannheim, Germany). In situ hybridization was performed as described previously [40].

\section{Results}

\section{Competence for metamorphosis was acquired around 8 dpf in P. pectinifera}

As in other echinoderm species, the transition from larval form to adult form proceeds via multiple steps in starfish. Development of the adult rudiment commences well before larvae acquire competence. Adult skeletogenesis was observed in penta-radial manner as early as 6 dpf, when development of brachiolar arms commences. Development of the brachiolar arms is tightly linked with the acquisition of competence. Murabe et al. indicated that adhesive papillae on the brachiolar arms sense environmental cues such as proper substrate for promoting metamorphosis [31]. When competent larvae attach to proper substrate, they initiate metamorphosis. The metamorphosis is observed as absorption of larval body and development of pentaradial juvenile body with primary podia (the precursor structures of tube feet), which occur within $24 \mathrm{~h}$ after settlement (Fig. 1).

Although development of the brachiolar arms commences at $6 \mathrm{dpf}$, we do not know when the competence to receive cues for this metamorphosis is acquired. Thus, we first sought to identify the stage at which $P$. pectinifera larvae become competent to metamorphose upon attachment to a substrate.

Here we examine the competence by testing whether larvae initiate metamorphosis upon attachment to coral sand taken from a tank of adult $P$. pectinifera. Note that we are not certain the coral sand was the most favorite substrate, and thus, larvae may initiate metamorphosis earlier when they attach to other substrates. We introduced coral sand to the wells of larvae of various ages (Fig. 2a) and counted the number of larvae that completed metamorphosis within $24 \mathrm{~h}$. Although larvae younger than $7 \mathrm{dpf}$ showed no response to the introduced substrate, some 8-dpf larvae $(0,6,0$ of 20 larvae from each batch) completed metamorphosis (Fig. 2b). The remaining larvae did not settle or metamorphose, but crawled around the substrate and attached repeatedly.
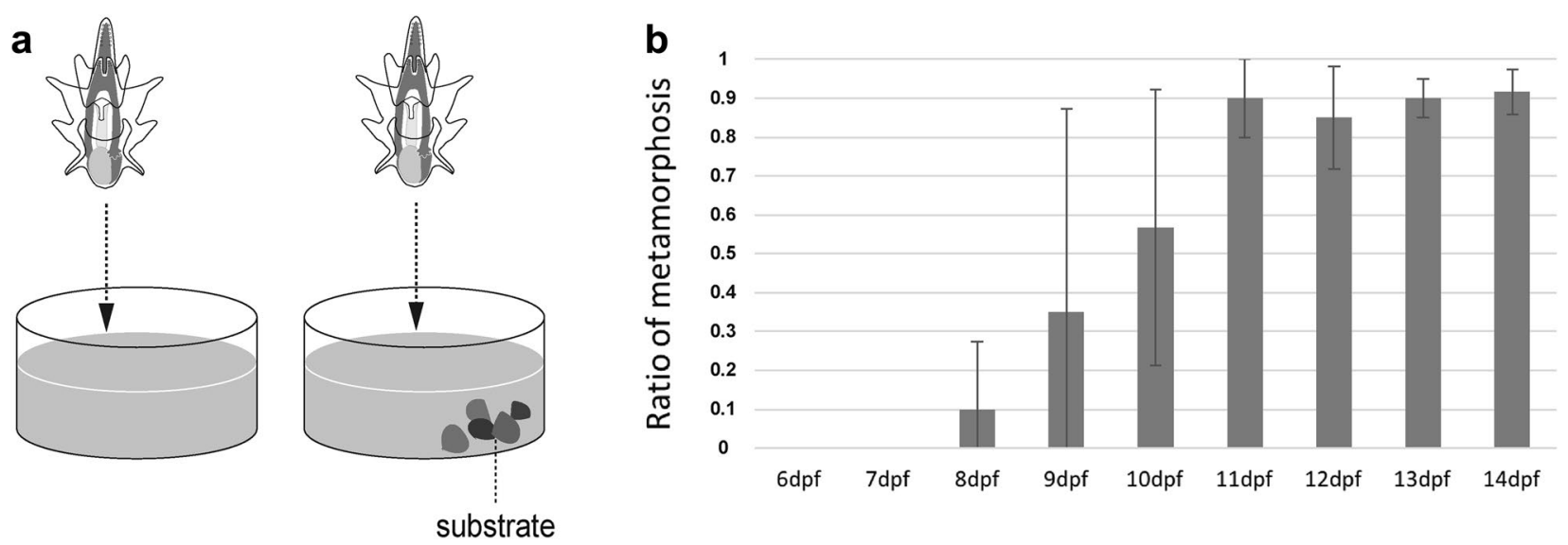

Fig. 2 Duration until P. pectinifera became competent. a Schematic experiment sets without or with substrate (left and right, respectively). Without external substrate, larva did not settle and complete metamorphosis. b Ratio of metamorphosis completion $24 \mathrm{~h}$ after adding substrate $(n=20$ from each three batch) 
This behavior was identified as characteristic prior to settlement and metamorphosis [31]. More than $85 \%$ of larvae older than $11 \mathrm{dpf}$ that were provided with substrate completed metamorphosis (Fig. 2b). These results indicate that larvae became competent to metamorphose upon attachment to coral sand at approximately $8 \mathrm{dpf}$. Of note, because larvae start feeding at $2 \mathrm{dpf}$, their growth and developmental rates depend on the nutrition level and are thus not uniform. Therefore, the reported age of the larvae is a rough estimate of the developmental stage.

\section{Exogenous RA treatments with competent $P$. pectinifera larvae induced metamorphosis}

We then investigated the function of RA signaling in the metamorphosis of $P$. pectinifera. Most competent larvae treated with $1 \mu \mathrm{M}$ all-trans RA at $14 \mathrm{dpf}$ showed larval body absorption and juvenile rudiment development with primary podia $(50,50$, and 47 of 50 larvae from each batch; Fig. 3b, e). Larval body absorption was observed immediately after RA treatment, and metamorphosis into a pentaradial juvenile body with primary podia was observed within $24 \mathrm{~h}$ (Fig. 3b, e). A similar effect was observed when larvae were treated with a lesser amount $(0.1 \mu \mathrm{M})$ of RA $(50,50$, and 40 of 50 larvae from each batch; Fig. 3a, d), whereas no larva showed any sign of larval body absorption or juvenile body development with the DMSO control $(0,0$, and 0 of 50 larvae from each batch; Fig. 3c).

These results indicate that all-trans RA has the potential to induce larval body absorption and juvenile rudiment development when added to competent larvae at 14 dpf.

\section{The effect of RA treatment on metamorphosis was limited after larvae became competent}

We investigated whether RA can also affect the timing of larval competence to respond to the metamorphosis cue. No larva treated with $1 \mu \mathrm{M}$ all-trans RA at 5-7 dpf commenced metamorphosis, as judged by larval body absorption and juvenile rudiment development (Fig. 3f). RA treatment of larvae younger than $8 \mathrm{dpf}$ had no apparent effect on morphology or behavior before $8 \mathrm{dpf}$. However, some of these larvae cultured in seawater with RA commenced metamorphosis at $8 \mathrm{dpf}$ (Fig. 3f). Regardless of when the RA treatment started, larvae responded to RA and metamorphosed at 8 or $9 \mathrm{dpf}$, which is comparable with the stage at which larvae acquire competence to metamorphose during normal development (Figs. 2, 3f). Most larvae that were treated with $1 \mu \mathrm{M}$ RA after $8 \mathrm{dpf}$ commenced metamorphosis within $24 \mathrm{~h}$ (Fig. 3f). These timelines are similar to those induced by a substrate. Above results indicate that RA does not affect the development of competence for metamorphosis, but rather functions as a cue to commence metamorphosis when added to competent larvae.

\section{RAR activation was required for the initiation of metamorphosis}

To examine whether RA functions as an internal mediator of signaling to a metamorphosis cue, we treated larvae with RO, known to be the selective RAR $\alpha$ antagonist (Fig. 4j). As shown previously, most 14-dpf larvae commenced metamorphosis when treated with $0.1 \mu \mathrm{M}$ or $1 \mu \mathrm{M}$ RA (Fig. 4a, c, e, g, i). We found that the presence of RO significantly suppressed metamorphosis rate $(P<0.001$, ANOVA, see Fig. 4, Additional file 5: Table S3) although effect of RA and interaction of RO and RA were not significant $(P=0.182$ and 0.633 , respectively, ANOVA). ANOVA revealed a significant effect of RO on the ratio of metamorphosed larvae $(P=0.182,0.000$, 0.632 and 0.108 in $\mathrm{RA}, \mathrm{RO}, \mathrm{RA}^{*} \mathrm{RO}$, and batch, respectively, ANOVA).

These results suggest that exogenous RA drives metamorphosis through binding to RAR. Therefore, we investigated whether RAR plays any role when larvae respond to a substrate and commence metamorphosis. We treated competent larvae with $1 \mu \mathrm{M}$ RO at $14 \mathrm{dpf}$. As shown above, more than $90 \%$ of 14-dpf larvae responded to the introduction of coral sand and commenced metamorphosis (Fig. 4k). However, when $1 \mu \mathrm{M}$ RO was added together with the substrate to 14-dpf larvae, metamorphosis was significantly inhibited $(P=0.000$ and 0.632 in $\mathrm{RO}$ and batch, respectively, ANOVA; Fig. 4k right, Additional file 6: Table S4). Interestingly, in contrast to metamorphosis, RO treatment did not affect the number of larvae attached to the substrate $(P=0.256$ and 0.058 in RO and batch, respectively, ANOVA; Fig. 4k left, Additional file 7: Table S5). These results are consistent with the idea that RAR functions as an internal mediator of the signaling cue for metamorphosis received by the adhesive papillae [31].

\section{Endogenous RA synthesis was essential for metamorphosis after settlement}

To assess whether endogenous RA synthesis was required for metamorphosis, we treated 14-dpf larvae with DEAB, a known inhibitor of Raldh (Fig. 4l). We cultured larvae in seawater with 100 or $300 \mu \mathrm{M}$ DEAB and coral sand as a substrate. DEAB-treated larvae crawled around the substrate surface, and most settled to the substrate with the brachiolar arms, as normal competent larvae do. The ratio of settled larvae in the DEAB treatments did not differ significantly $(P=0.728$, ANOVA; Fig. 4m left, Additional file 8: Table S6), although significant difference was observed among batches $(P=0.010$ in batch, ANOVA). However, DEAB significantly inhibited 


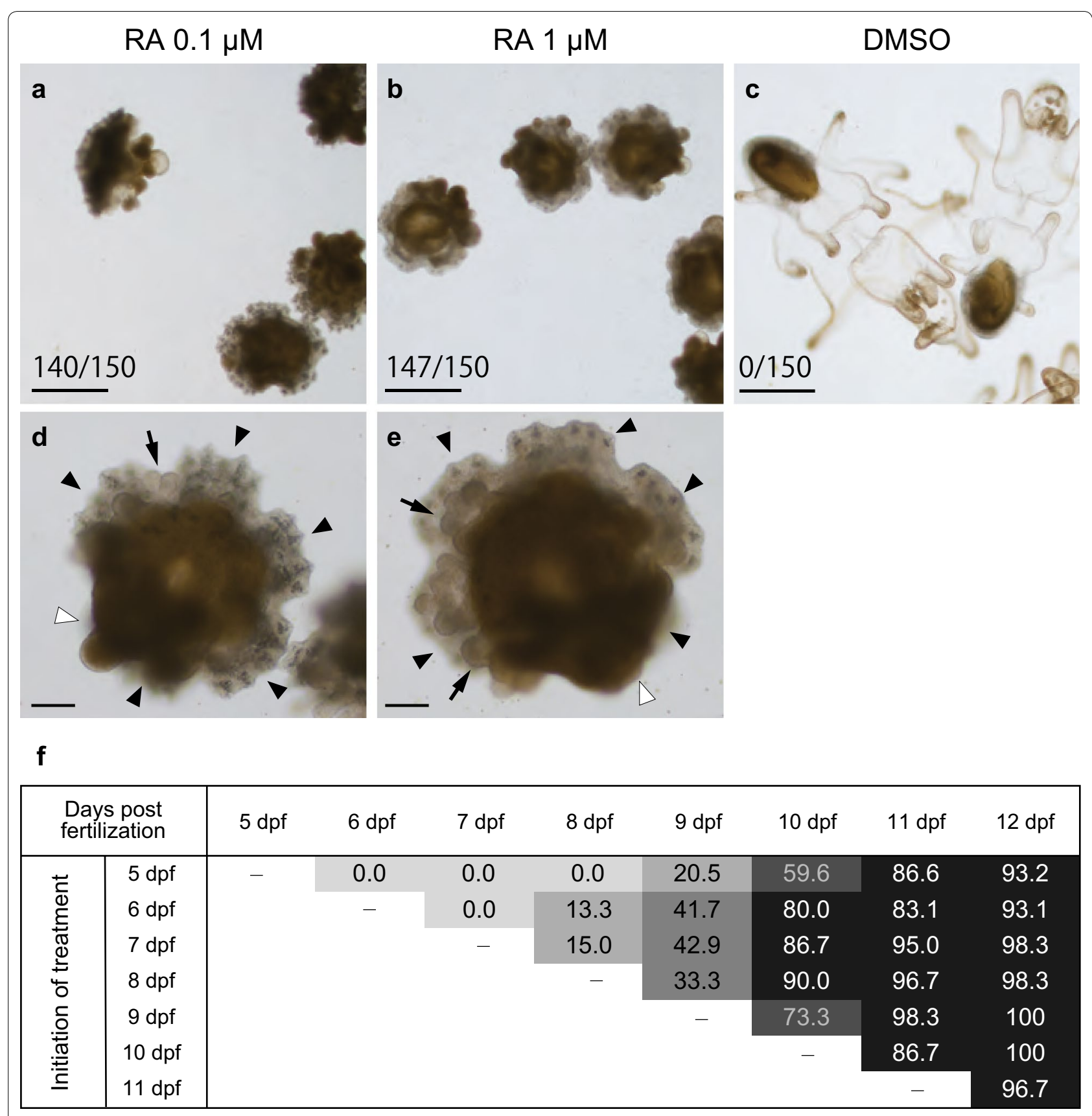

Fig. 3 Effects on metamorphosis by all-trans RA or DMSO treatments in P. pectinifera. a-e Metamorphosis induction of 14-dpf larvae by exogenous all-trans RA (0.1 or $1 \mu \mathrm{M}$ ) treatment ( $n=50$ from each three batch). $\mathbf{a}, \mathbf{b}, \mathbf{d}, \mathbf{e}$ Induced juvenile of $96 \mathrm{~h}$ after RA $0.1,1 \mu \mathrm{M}$ treatment, respectively. $\mathbf{c}$ DMSO-treated larvae ( $96 \mathrm{~h}$ after treatment). Arrow heads indicate developed juvenile rudiment, white arrowheads point out absorbed larval body, and arrows show primary podia. The scale bars in $\mathbf{a}-\mathbf{c}, \mathbf{d}-\mathbf{e}$ display $250,50 \mu \mathrm{m}$, respectively. The number in figure show the ratio of metamorphosed larvae/treated larvae. f Temporal effects on metamorphosis induction of all-trans RA (1 $\mu \mathrm{M})$. Numbers in figure show percentage of metamorphosed specimen ( $n=20$ from each three batch in each treatment) and background colors are depended on their percentage and divided into five darkness levels, meaning 0, 0-25, 25-50, 50-75 and 75-100\%. Vertical axis indicates days after treatment and horizontal axis indicates days after fertilization

metamorphosis after settlement. Significance of DEAB was found in metamorphosis $(P=0.000$ and 0.001 in DEAB and batch, respectively, ANOVA; Fig. $4 \mathrm{~m}$ right,
Additional file 9: Table S7). The metamorphosis rate was slightly lower in the $100 \mu \mathrm{M}$ DEAB treatment than in the DMSO treatment. Inhibition of $300 \mu \mathrm{M}$ DEAB 


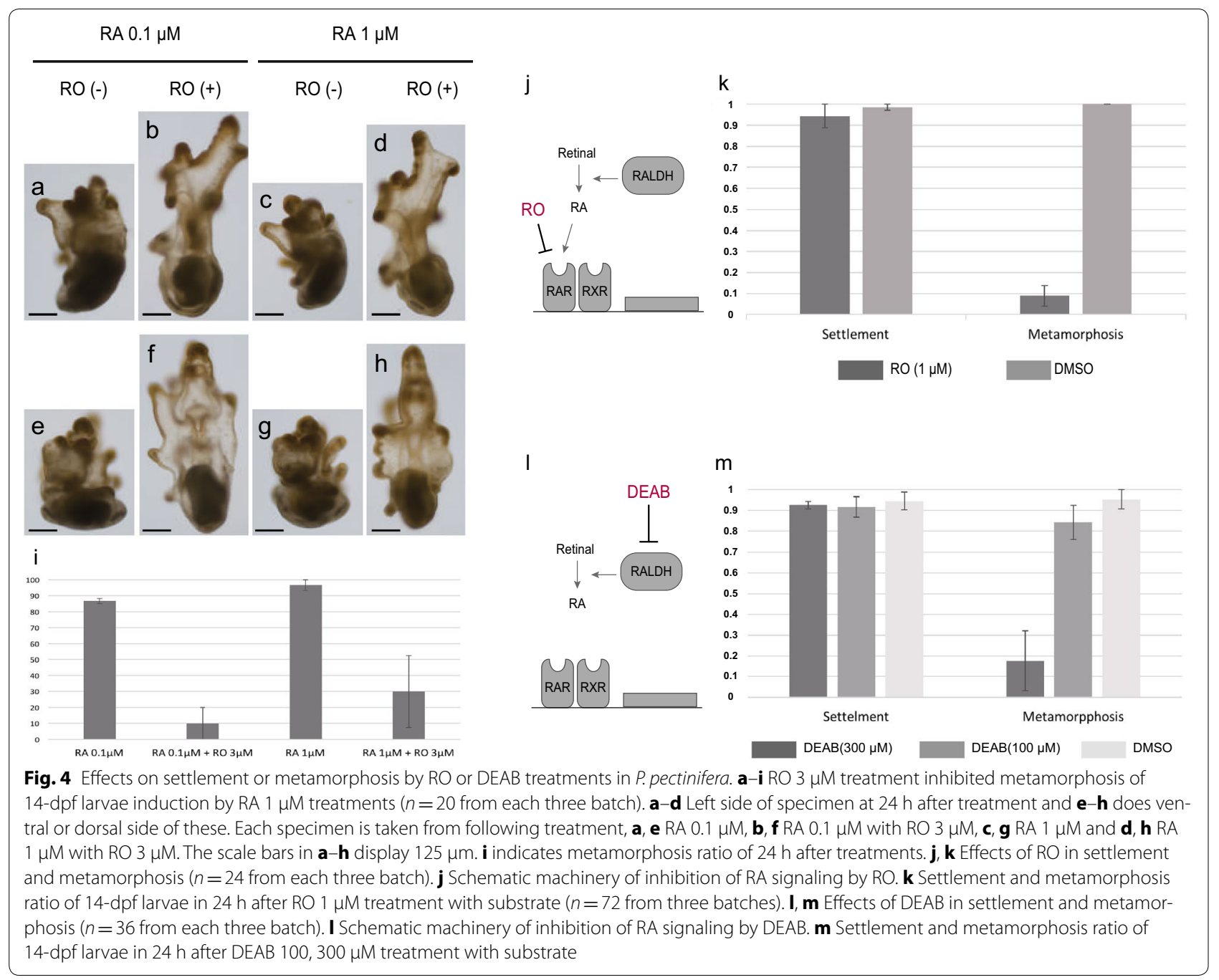

was significantly effective with DMSO and $100 \mu \mathrm{M}$ DEAB treatments $(P=0.000$ and $P=0.000$, Tukey's test, respectively), although there was no significant difference between the latter two in metamorphosis $(P=0.624$, Tukey's test). These results indicate that endogenous RA synthesis was essential for the commencement of metamorphosis after receipt of the signaling cue by the adhesive papillae, whereas it was not essential for settlement on the substrate.

Temporal and spatial expression patterns of genes related to RA signaling in larvae

The results reported above suggest that RA signaling functions as an endogenous mediator of a metamorphosis cue upon settlement. To support this idea, we surveyed RA signaling genes in our transcriptome data for P. pectinifera. We investigated their orthologues by construction of phylogenetic trees (Additional file 1: Fig. S1, Additional file 2: Fig. S2). We identified three raldh genes in our transcriptome data: raldha, raldhb and raldhc (Additional file 1: Fig. S1). Single genes for rar and $r x r$ were also identified from our transcriptome data (Additional file 2: Fig. S2).

We examined the spatial expression patterns of raldha, raldhb, raldhc, rar, and rxr by whole mount in situ hybridization in larvae of various stages (Fig. 5). P. pectinifera larvae became competent to metamorphose after $8 \mathrm{dpf}$, as shown previously. The expression of raldha, raldhb, and raldhc was detected in the foregut, midgut, and hindgut of larvae as young as $6 \mathrm{dpf}$, but no specific expression of rar or $r x r$ was observed (Fig. 5a-e). In 9-dpf larvae that acquired competence, overlapping expression of raldha, rar, and rxr was detected in the juvenile rudiment and the epidermis around the brachiolar arms, whereas expression of raldhb and raldhc was restricted to the foregut, midgut and hindgut (Fig. $5 f-j$ ). Similar expression patterns were observed in 14-dpf larvae that did not receive environmental cues for metamorphosis, 


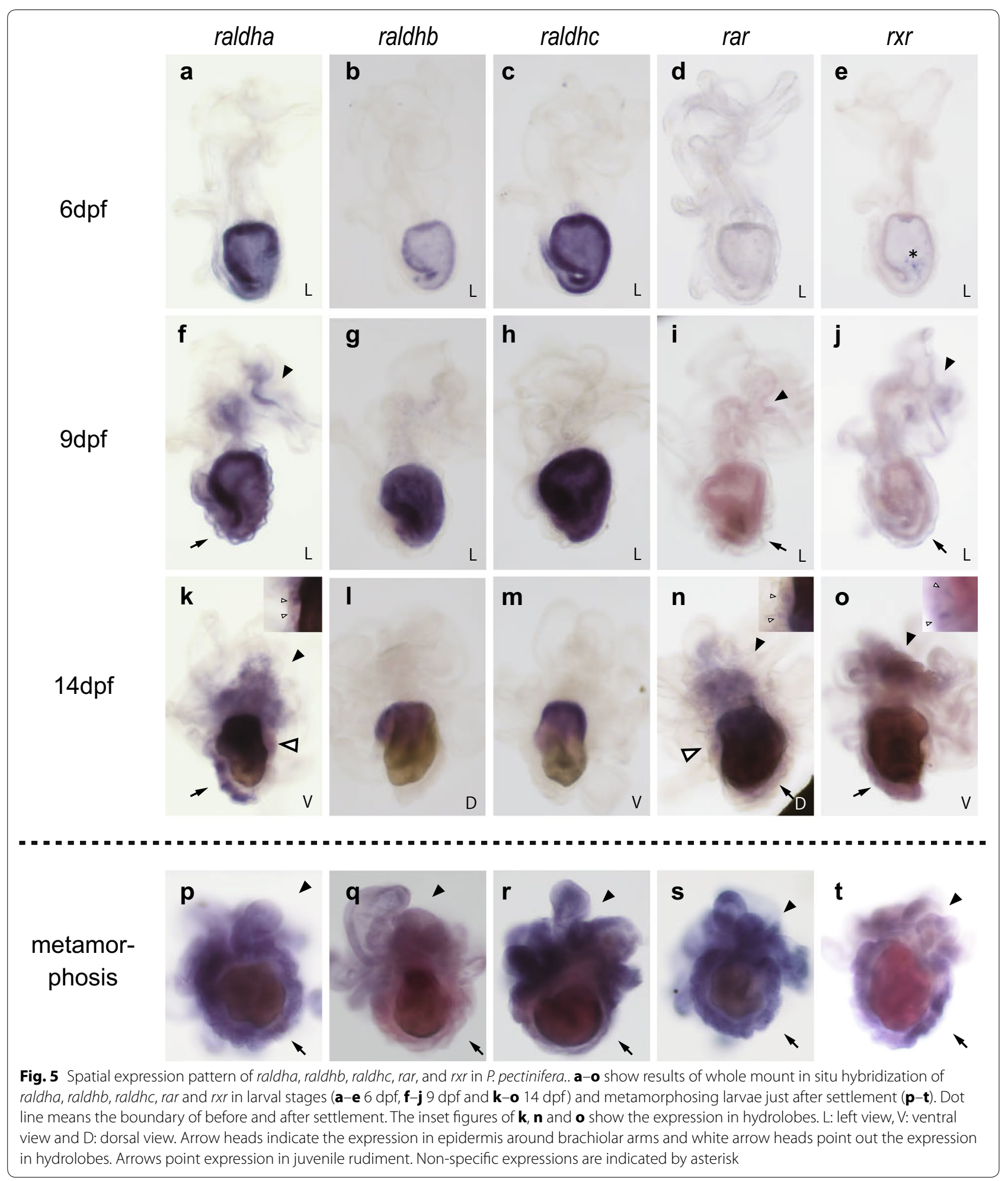


except that the expression of raldha, rar and rxr was also detected in the hydrolobes (Fig. 5k-o). In metamorphosing larvae after settlement, all genes that we examined were expressed in the whole epidermis of the larval body and in the juvenile rudiment (Fig. 5q-t). These expression patterns are consistent with our hypothesis that RA functions as an endogenous mediator of environmental cues, although an additional regulator seems to be required to activate RA signaling upon receiving cues, as discussed later in this paper.

\section{Discussion}

RA signaling acts as a regulator of starfish metamorphosis The life cycle transition from larva to juvenile in echinoderms occurs via two key steps. First, larvae acquire competence to metamorphose. Second, after receiving an environmental cue, metamorphosis proceeds with the absorption of the larval body and development of the pentaradial juvenile body. We present evidence that RA signaling functions as an endogenous mediator of environmental cues received upon attachment to a substrate in starfish (Fig. 6). Metamorphosis was suppressed by the inhibition of two distinct steps of RA signaling (Fig. 4): RA synthesis from retinal (by DEAB) and the reception of RA by RAR (by RO). We have not directly shown that $\mathrm{RA}$ is present in starfish larvae. Identification of three raldh genes, and the overlapping expression of one of them with rar and $r x r$ in competent larvae (Fig. 5), however, strongly supports the idea that RA functions as an endogenous signaling molecule in starfish development.

Of note, the expression of raldhs, rar, and rxr was observed well before competent larvae received environmental cues (Fig. 5a-o). These expression patterns suggest the presence of another regulatory mechanism that controls the onset of RA signaling upon attachment. Murabe et al. indicated that environmental cues were received by adhesive papillae on the brachiolar arms [31]. Thus, the onset of RA signaling may be regulated by something in the adhesive papillae, likely through the nervous system. NO and HA are candidates for this regulator, as both have inhibitory effects on the onset of metamorphosis in sea urchins [11, 17, 24, 25]. Although no evidence of NO signaling or HA signaling has been reported for starfish, further studies on the crosstalk of these signals with RA signaling will facilitate a comprehensive understanding of the molecular regulatory mechanism of echinoderm metamorphosis.
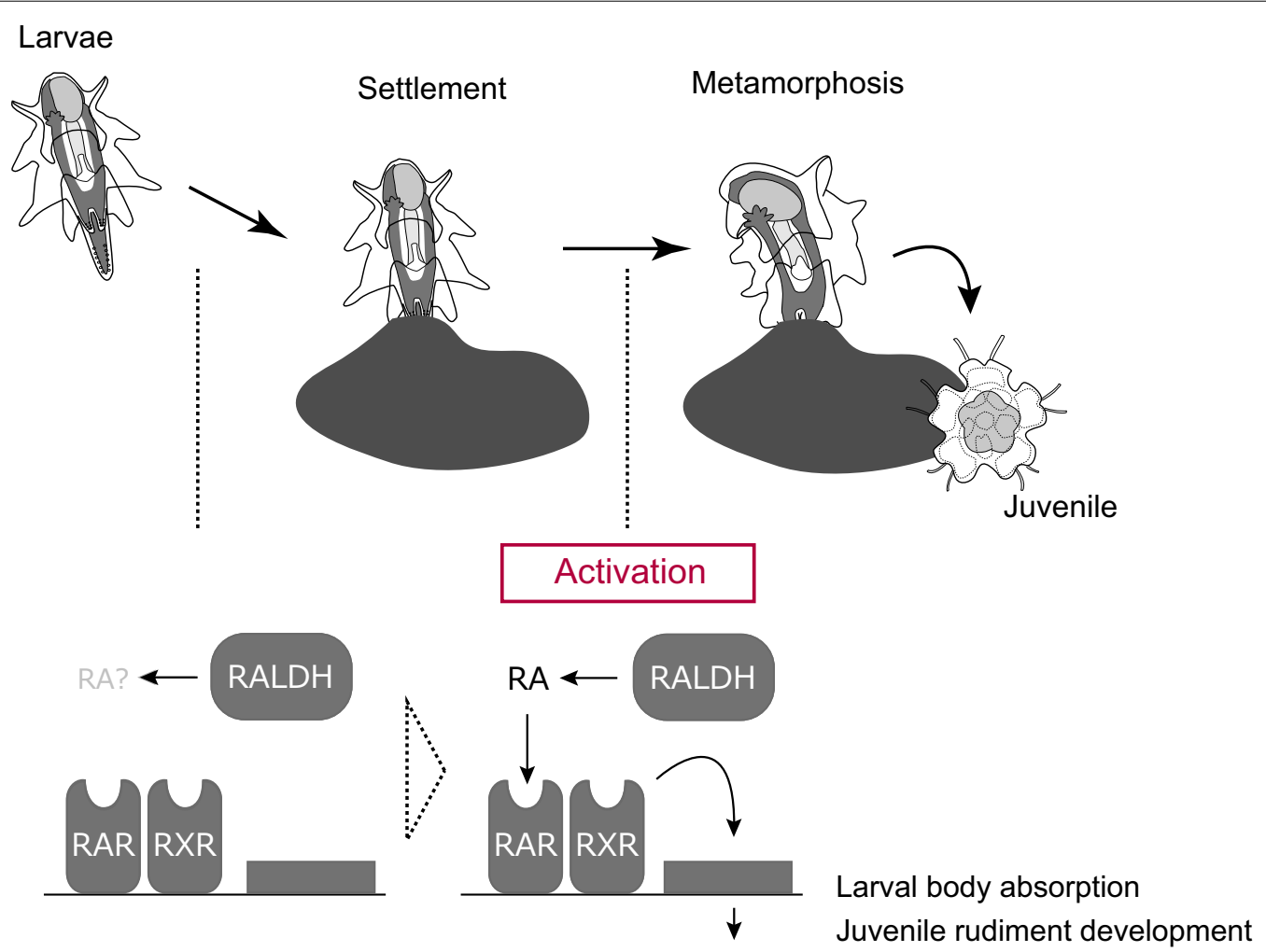

Fig. 6 Schematic RA signaling function as regulator of metamorphosis. RA signaling activation is induced by receiving environmental cue with adhesive papillae of brachiolar arms and commences metamorphosis process 
The role of the nuclear receptor/RXR heterodimer in metamorphosis regulation is evolutionarily conserved RA signaling mediated by a RAR/RXR heterodimer receptor plays a critical role in the development of the unique body plans of chordates, such as the anteriorposterior patterning of the central nervous system [1, $2,7]$. Therefore, the ancestral role of RA signaling has attracted the interest of several zoologists [5, 6]. Our findings suggest that RA signaling plays a role in the regulation of metamorphosis in ancestral deuterostomes. During the evolution of ancestral chordates, RA signaling may have been co-opted for the patterning of the chordate body plan via the recruitment of Hox genes as regulators. Further investigation of RA signaling in nonchordate deuterostomes may shed new light on the evolution of the vertebrate body plan.

The transition of distinct body plans through metamorphosis is widely observed in metazoans [11, 41], and several recent studies have revealed that conserved molecular regulatory mechanisms underlie metamorphosis $[10,11,42]$. Molecular mechanisms for metamorphosis in amphibians and insects have been investigated in detail [12-16]. In both taxa, metamorphosis is regulated by hormones received by receptors ( $\mathrm{TH}$ for amphibians and an ecdysone receptor for insects), which make a heterodimer with RXR. A recent study by Fuchs indicated that RXR is also involved in cnidarian metamorphosis [10]. Exogenous treatment with RA was shown to induce the metamorphic process of strobilation. Here, we present evidence that RA signaling is also involved in starfish metamorphosis, and provide additional evidence that components of RA signaling are conserved in this metamorphosis. However, amphibians and insects use different hormones for signaling, and RXR makes heterodimers with different counterparts accordingly. The suggested existence of a common ligand in cnidarians and starfish is thus intriguing and may reflect the ancestral molecular mechanism for metazoan metamorphosis. This hypothesis will be tested by further investigation of metamorphosis in various taxa, such as sea urchins, annelids and molluscs.

\section{Conclusions}

We demonstrated that RA signaling performs as the regulator of metamorphosis process in echinoderm (starfish). Considering RA is also core element of metamorphosis regulation in basal group of metazoan (cnidarian), our results suggest that ancestral function of RA signaling is involved in metamorphosis.

\section{Additional files}

Additional file 1: Figure S1. Phylogenic tree of Raldh (=Aldh1a, Aldehyde hydrogenase $1 \mathrm{alfa}$ ) and Aldh2 (Aldehyde hydrogenase 2) subfamily. The phylogenic tree was constructed by RAxML. raldhs of starfish made sister grouping with hemichordate aldh 1 as. Numbers at node show bootstrap value. The selected amino acid substation model was $L G+F+G$.

The process to construct was described in material and method and the set of sequences is provided in Additional file 10: Supplementary dataset 1. Abbreviations of species were referred following, $\mathrm{Hs}$; Homo sapiens (Human), Mm; Mus musculus (Mouse), Xt; Xenopus tropicalis (Western clawed frog), Dr; Danio rerio (Zebrafish), Bf; Branchiostoma floridae (Amphioxus), Ci; Ciona intestinalis (Transparent sea squirt), Sk; Saccoglossus kowalevskii (Acorn worm), Sp; Strongylocentrotus purpuratus (Purple sea urchin), Pp; Patiria pectinifera.

Additional file 2: Figure S2. Phylogenic tree of RAR, RXR and THR (Thyroid hormone receptor). The phylogenic tree was constructed by RAxML. The selected amino acid substation model was $L G+F+G$. The set of sequences is provided in Additional file 11: Supplementary dataset 2. Abbreviations of species were referred following, $\mathrm{Hs}$; Homo sapiens (Human), Mm; Mus musculus (Mouse), Xt; Xenopus tropicalis (Western clawed frog), Dr; Danio rerio (Zebrafish), Bf; Branchiostoma floridae (Amphioxus), Pm; Polyandrocarpa misakiensis (Tunicate), Bl; Branchiostoma lanceolatum (Amphioxus), Ci; Ciona intestinalis (Transparent sea squirt), Sk; Saccoglossus kowalevskii (Acorn worm), Sp; Strongylocentrotus purpuratus (Purple sea urchin), Pp; Patiria pectinifera, Dm; Drosophila melanogaster (Fruit fly), Rc; Reishia clavigera (Sea snail), Ls; Lymnaea stagnalis (Great pond snail), Tc; Tripedalia cystophora (Jellyfish).

Additional file 3: Table S1. Accession numbers of the gene used for construction of phylogenic tree. Amino acid sequences to construct tree were obtained from Uniprot or Echinobase (http://www.echinobase.org/ Echinobase/), Genbank.

Additional file 4: Table S2. Sequences of primer for amplification of raldha, raldhb, raldhc, rar and rxr. We used 40-bp reverse primers including a 20-bp T3 promoter sequence to synthesize Dig-labelled RNA probes for in situ hybridization. Bold characters mean consensus sequence for T3 promoter.

Additional file 5: Table S3. Number of metamorphosed/treated larvae of each batch in $\mathrm{RO}+\mathrm{RA}$ treatment experiment.

Additional file 6: Table S4. Number of metamorphosed/settled larvae of each batch in RO $1 \mu \mathrm{M}$ or DMSO treatment.

Additional file 7: Table S5. Number of settled larvae/treated larvae of each batch in RO $1 \mu \mathrm{M}$ or DMSO treatment.

Additional file 8: Table S6. Number of settled/treated larvae of each batch in DEAB 100, $300 \mu \mathrm{M}$ or DMSO treatment.

Additional file 9: Table S7. Number of metamorphosed/settled larvae of each batch in DEAB 100, $300 \mu \mathrm{M}$ or DMSO treatment.

Additional file 10: Supplementary dataset 1. The dataset for phylogenetic analysis of Raldh and Aldh2.

Additional file 11: Supplementary dataset 2. The dataset for phylogenetic analysis of RAR, RXR and THR.

\section{Authors' contributions}

SY, YM, and HW contributed to the design of the experiments. SY and YM collected the samples. SY performed the experiments. SY and MH analyzed the data. All authors wrote the manuscript. All authors read and approved the final manuscript. 


\section{Acknowledgements}

We thank Ei-ichi Okumura, Ritsu Kuraishi, and Akihito Wanishi for help in collecting P. pectinifera. We also thank the Ibaraki Prefectural Oarai Aquarium for providing the seawater for culturing adult starfish.

\section{Competing interests}

The authors declare that they have no competing interests.

\section{Availability of data and materials}

The sequences of starfish gene (raldha, raldhb, raldhc, rar, and $r x$ r) were deposited to the DNA Data Bank of Japan (DDBJ; LC379260, LC379261 LC379262, LC379258 and LC379259, respectively). The dataset for phylogenetic analysis was supplied in Additional files 10 and 11. The raw reads of transcriptome are available from the DDBJ Sequence Reads Archives (DRA006662).

\section{Consent for publication}

Not applicable.

\section{Ethics approval and consent to participate}

Not applicable.

\section{Funding}

This work was supported by JSPS KAKENHI Grant No. 15KT0074.

\section{Publisher's Note}

Springer Nature remains neutral with regard to jurisdictional claims in published maps and institutional affiliations.

Received: 6 February 2018 Accepted: 12 April 2018

Published online: 21 April 2018

\section{References}

1. Gutierrez-Mazariegos J, Schubert M, Laudet V. Evolution of retinoic acid receptors and retinoic acid signaling. Subcell Biochem. 2014;70:55-73.

2. Rhinn M, Dolle P. Retinoic acid signalling during development. Development. 2012;139(5):843-58

3. Marlétaz F, Holland LZ, Laudet V, Schubert M. Retinoic acid signaling and the evolution of chordates. Int J Biol Sci. 2006:2(2):38.

4. Escriva H, Delaunay F, Laudet V. Ligand binding and nuclear receptor evolution. BioEssays. 2000;22(8):717-27.

5. Cañestro C, Postlethwait JH, Gonzàlez-Duarte R, Albalat R. Is retinoic acid genetic machinery a chordate innovation? Evol Dev. 2006:8(5):394-406.

6. Albalat $\mathrm{R}$. The retinoic acid machinery in invertebrates: ancestral elements and vertebrate innovations. Mol Cell Endocrinol. 2009:313(1-2):23-35.

7. Campo-Paysaa F, Marletaz F, Laudet V, Schubert M. Retinoic acid signaling in development: tissue-specific functions and evolutionary origins. Genesis. 2008;46(11):640-56.

8. Kuno S, Kawamoto M, Okuyama M, Yasumasu I. Outgrowth of pseudopodial cables induced by all-trans retinoic acid in micromere-derived cells isolated from sea urchin embryos. Dev Growth Differ. 1999;41(2):193-9.

9. Sciarrino S, Matranga V. Effects of retinoic acid and dimethylsulfoxide on the morphogenesis of the sea urchin embryo. Cell Biol Int. 1995;19(8):675-80.

10. Fuchs B, Wang W, Graspeuntner S, Li Y, Insua S, Herbst EM, Dirksen P, Bohm AM, Hemmrich G, Sommer F, et al. Regulation of polyp-to-jellyfish transition in Aurelia aurita. Curr Biol. 2014:24(3):263-73.

11. Heyland A, Moroz LL. Signaling mechanisms underlying metamorphic transitions in animals. Integr Comp Biol. 2006;46(6):743-59.

12. Laudet $\mathrm{V}$. The origins and evolution of vertebrate metamorphosis. Curr Biol. 2011;21(18):R726-37.

13. Buszczak M, Segraves WA. Drosophila metamorphosis: the only way is USP? Curr Biol. 1998;8(24):R879-82.

14. Thummel CS. From embryogenesis to metamorphosis: the regulation and function of Drosophila nuclear receptor superfamily members. Cell. 1995:83(6):871-7.

15. Buszczak M, Segraves WA. Insect metamorphosis: out with the old, in with the new. Curr Biol. 2000;10(22):R830-3.

16. Brown DD, Cai L. Amphibian metamorphosis. Dev Biol. 2007;306(1):20-33.
17. Bishop CD, Brandhorst BP. Development of nitric oxide synthase-defined neurons in the sea urchin larval ciliary band and evidence for a chemosensory function during metamorphosis. Dev Dyn. 2007;236(6):1535-46.

18. Bishop CD, Brandhorst BP. NO/CGMP signaling and HSP90 activity represses metamorphosis in the sea urchin Lytechinus pictus. Biol Bull. 2001;201(3):394-404

19. Chino Y, Saito M, Yamasu K, Suyemitsu T, Ishihara K. Formation of the adult rudiment of sea urchins is influenced by thyroid hormones. Dev Biol. 1994;161(1):1-11.

20. Heyland A, Price DA, Bodnarova-Buganova M, Moroz LL. Thyroid hormone metabolism and peroxidase function in two non-chordate animals. J Exp Zool B Mol Dev Evol. 2006;306(6):551-66.

21. Johnson LG. Stage-dependent thyroxine effects on sea urchin development. N Z J Mar Freshw Res. 1998;32(4):531-6.

22. Miller AE, Heyland A. lodine accumulation in sea urchin larvae is dependent on peroxide. J Exp Biol. 2013:216(Pt 5):915-26.

23. Naidenko TK. Induction of metamorphosis of two species of sea urchin from Sea of Japan. Mar Biol. 1996;126(4):685-92.

24. Swanson RL, Williamson JE, De Nys R, Kumar N, Bucknall MP, Steinberg PD. Induction of settlement of larvae of the sea urchin Holopneustes purpuras cens by histamine from a host alga. Biol Bull. 2004;206(3):161-72.

25. Sutherby J, Giardini J-L, Nguyen J, Wessel G, Leguia M, Heyland A. Histamine is a modulator of metamorphic competence in Strongylocentrotus purpuratus (Echinodermata: Echinoidea). BMC Evol Biol. 2012;12(1):14.

26. Heyland A, Hodin J. Heterochronic developmental shift caused by thyroid hormone in larval sand dollars and its implications for phenotypic plasticity and the evolution of nonfeeding development. Evolution. 2004;58(3):524-38.

27. Heyland A, Reitzel AM, Hodin J. Thyroid hormones determine developmental mode in sand dollars (Echinodermata: Echinoidea). Evol Dev. 2004:6(6):382-92.

28. Johnson LG, Cartwright CM. Thyroxine-accelerated larval development in the crown-of-thorns starfish, Acanthaster planci. Biol Bull. 1996;190(3):299-301.

29. Rowley R. Settlement and recruitment of sea urchins (Strongylocentrotus spp) in a sea-urchin barren ground and a kelp bed: are populations regulated by settlement or post-settlement processes? Mar Biol. 1989:100(4):485-94.

30. Koga H, Matsubara M, Fujitani H, Miyamoto N, Komatsu M, Kiyomoto $\mathrm{M}$, Akasaka K, Wada H. Functional evolution of Ets in echinoderms with focus on the evolution of echinoderm larval skeletons. Dev Genes Evol. 2010;220(3-4):107-15.

31. Murabe N, Hatoyama H, Komatsu M, Kaneko H, Nakajima Y. Adhesive papillae on the brachiolar arms of brachiolaria larvae in two starfishes, Asterina pectinifera and Asterias amurensis, are sensors for metamorphic inducing factor(s). Dev Growth Differ. 2007;49(8):647-56.

32. Team RC. R: a language and environment for statistical computing. Vienna: R Foundation for Statistical Computing; 2017

33. Logan M. Biostatistical design and analysis using R: a practical guide. Chichester: Wiley; 2011.

34. Zar JH. Biostatistical analysis. 4th ed. Englewood Cliffs: Prentice Hall; 1998

35. Patel RK, Jain M, Liu Z. NGS QC toolkit: a toolkit for quality control of next generation sequencing data. PLoS ONE. 2012;7(2):e30619.

36. Grabherr MG, Haas BJ, Yassour M, Levin YZ, Thompson DA, Amit I, Adiconis X, Fan L, Raychowdhury R, Zeng Q, Chen Z, Mauceli E, Hacohen N, Gnirke A, Rhind N, di Palma F, Birren BW, Nusbaum C, Lindblad-Toh K, Friedman N, Regev A. Full-length transcriptome assembly from RNA-Seq data without a reference genome. Nat Biotechnol. 2011:29(7):644-52.

37. Katoh K, Rozewicki J, Yamada KD. MAFFT online service: multiple sequence alignment, interactive sequence choice and visualization. Brief Bioinform. 2017. https://doi.org/10.1093/bib/bbx108.

38. Capella-Gutiérrez S, Silla-Martínez JM, Gabaldón T. trimAl: a tool for automated alignment trimming in large-scale phylogenetic analyses. Bioinformatics. 2009;25(15):1972-3.

39. Stamatakis A. RAxML version 8: a tool for phylogenetic analysis and postanalysis of large phylogenies. Bioinformatics. 2014;30(9):1312-3.

40. Morino Y, Koga H, Tachibana K, Shoguchi E, Kiyomoto M, Wada H. Heterochronic activation of VEGF signaling and the evolution of the skeleton in echinoderm pluteus larvae. Evol Dev. 2012;14(5):428-36.

41. Degnan SM, Degnan BM. The initiation of metamorphosis as an ancient polyphenic trait and its role in metazoan life-cycle evolution. Philos Trans R Soc Lond B Biol Sci. 2010;365(1540):641-51.

42. Heyland A, Moroz LL. Cross-kingdom hormonal signaling: an insight from thyroid hormone functions in marine larvae. J Exp Biol. 2005;208(Pt 23):4355-61. 